\title{
Preventive Maintenance Strategy of Environmental Test Chamber Based on Particle Swarm Optimization Algorithm
}

\author{
Xianwen Zhou ${ }^{1}$, Chaoyang Gu${ }^{1}$, Yuyu Sun ${ }^{2}$, Chengjing Han ${ }^{3}$, Wei Gu${ }^{1}$, Wangqiang Niu ${ }^{1}$ \\ ${ }^{1}$ Key Laboratory of Transportation Technology and Control Engineering, Shanghai Maritime \\ University, Shanghai 201306, China \\ ${ }^{2}$ Jiangsu Automation Research Institute, Lianyungang 222021, China \\ ${ }^{3}$ Shanghai Sizhong Experimental Equipment Co., Ltd, Shanghai 201615, China
}

Received: July 20, 2021. Revised: December 7, 2021. Accepted: January 13, 2022. Published: January 15, 2022.

\begin{abstract}
With the development of various physical industries, people pay more attention to reliability tests and test equipment. To solve the problem of making maintenance strategy of an environmental test chamber for reliability test, a periodic preventive maintenance strategy based on RCM(Reliability Centre Maintenance) is proposed. Firstly, a multi-objective optimization model of reliability and maintenance cost is established by combining reliability theory and life distribution theory, and two objectives of equipment reliability and maintenance cost are considered. Secondly, the actual environmental test chamber fault maintenance data is analyzed, and it is found the fault distribution meets the dual parameter Weibull. Finally, the particle swarm optimization algorithm is used to solve the multi-objective model optimization, and a series of Pareto optimal solutions are obtained, that is, the number of maintenance times and the corresponding time interval in the operation cycle of the environmental test chamber, and these solutions might be good references for maintenance management personnel.
\end{abstract}

Keywords-Environmental test chamber, Reliabilitymaintenance cost model, Fault distribution, Particle Swarm Optimization.

\section{INTRODUCTION}

$\mathrm{T}_{\mathrm{e}}^{\mathrm{h}}$ environmental test chamber is a kind of box-type equipment for reliability tests, which is mostly used to test the reliability and stability performance parameters of various products under the setting conditions of temperature and humidity [1]. Chaoyang et al. applied and analyzed the reliability model of the environmental test chamber[2]. As important equipment of product reliability testing, the reliability of the environmental test chamber itself needs to have high stability. For the same environmental test chamber, different maintenance strategies will produce different cost and reliability index changes, so it is necessary to optimize the maintenance decision.

The maintenance of equipment is generally defined as the activity to make the equipment resume operation after the equipment stops operation due to failure [3]. Yonghu et al. put forward a preventive maintenance strategy of equipment based on particle swarm optimization [4]; Maintenance optimization based on reliability is proposed by Chen et al.[5]; Xiao Yu et al. propose an equipment maintenance strategy model with the maximum success probability as the maintenance goal [6]. Most studies assume that the fault distribution satisfies the sharp distribution of the two-parameter Weibull distribution, and do not calculate the fault distribution with the actual data.

This paper studies the maintenance strategy of the environmental test chamber. As an important carrier of reliability tests, the environmental test chamber must maintain a relatively high-reliability level to meet the test requirements. Therefore, the maintenance strategy of the environmental test chamber is considered to adopt reliability-centered maintenance RCM [7]. At the same time, the environmental test chamber will not be interrupted in operation, so it is necessary to plan the maintenance time and select the non-working time for preventive maintenance (PM). This study uses a reliability-centered maintenance plan and sequential preventive maintenance plan to construct a multi-objective maintenance strategy model with comprehensive consideration of reliability and cost factors [8]. The Pareto solution of a maintenance strategy is obtained by particle swarm optimization.

By analyzing the current research direction of maintenance strategy, it can be found that under the requirements of high reliability, there are few relevant studies on how to effectively solve the contradiction between serious equipment loss caused by high-intensity use and maintaining high equipment integrity rate. Combined with the actual task, it is proposed to strengthen preventive maintenance, and scientifically predict the optimal preventive maintenance times and time interval of equipment in the service period by establishing a mathematical model, to improve the equipment support efficiency.

\section{MAINTENANCE STRATEGY AND OPTIMIZATION MODEL}

\section{A. Reliability model of maintenance plan}

Considering the reliability of the environmental test chamber, in the case of sequential preventive maintenance, it is assumed that the working time of the environmental test chamber is $[0, T]$. Maintenance intervals and times need to be considered to ensure that the reliability and cost of the environmental test chamber meet the expected requirements [6].

The time interval of preventive maintenance is defined as $t_{i}$. The purpose of this study is to determine the number of 
maintenance $N$ and the time interval $t_{i}$ between each maintenance. To facilitate the model construction, this paper assumes that the failure rate of the environmental test chamber after maintenance is consistent with that before maintenance, and the maintenance time is ignored.

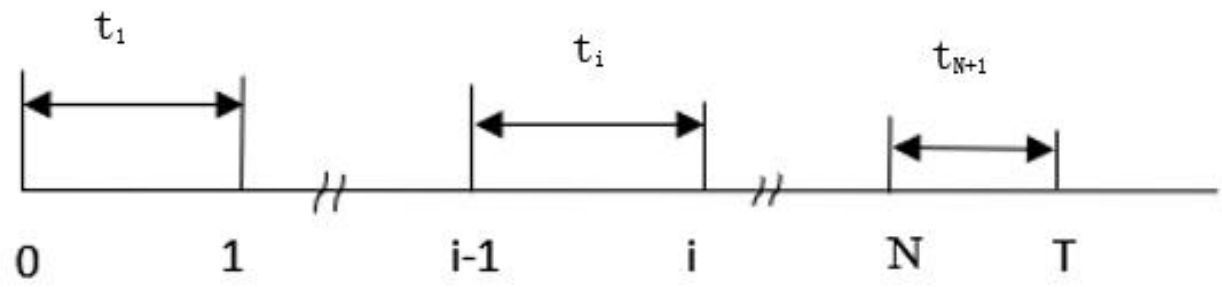

Fig. 1 Schematic diagram of maintenance plan

Sequential preventive maintenance is based on the determined time interval. Because the determined time intervals are different, the reliability of the total operation time $(0, T)$ is studied. The average reliability is calculated as follows:

$$
\overline{R(t)}=\frac{\int_{0}^{T} R(t) d t}{T}
$$

According to the reliability theory, the reliability function of the environmental test chamber in each preventive maintenance interval is as follows:

$$
R_{i}(t)=\exp \left(-\int_{0}^{t} \lambda_{i}(t) d t\right)
$$

where $\lambda(t)$ is a function of failure rate; The reliability of the environmental test chamber always changes according to the change of maintenance time interval, so the average reliability of the whole time cycle is used to represent the overall reliability of the equipment:

$$
R_{a}(t)=\frac{\sum_{i=1}^{N+1} \int_{0}^{t_{i}} R_{i}(t) d t}{\sum_{i=1}^{N+1} t_{i}}
$$

The reliability optimization model is as follows:

Find:

$$
N, t_{i}
$$

Max:

$$
R\left(N, t_{i}\right)=\frac{\sum_{i=1}^{N+1} \int_{0}^{t_{i}} \exp \left(-\int_{0}^{t} \lambda(t) d t\right) d t}{\sum_{i=1}^{N+1} t_{i}}
$$

s.t.

$$
\sum_{i=1}^{N+1} t_{i} \leq T
$$

\section{B. Maintenance planning cost model}

During the whole period of equipment operation $[0, \mathrm{~T}]$, the maintenance cost consists of the following parts. Minimum maintenance cost within maintenance interval:

$$
C_{\mathrm{min}}=\sum_{i=1}^{N+1} \int_{0}^{t_{i}} c_{c m} \bullet \lambda(t) d t
$$

Unscheduled preventive maintenance cost:

$$
C_{2}=\sum_{i=1}^{N} c_{p m}\left(t_{i}\right)
$$

The maintenance cost of the equipment in the whole operation process is as follows:

$C=C_{\text {min }}+C_{2}+C_{3}=\sum_{i=1}^{N+1} \int_{0}^{t_{i}} c_{c m} \bullet \lambda(t) d t+\sum_{i=1}^{N} c_{p m}\left(t_{i}\right)+\sum_{i=1}^{N} c_{p t}$

\section{C.Multi-objective model of maintenance planning}

During the service period of the environmental test chamber, the scientific implementation of preventive maintenance can effectively reduce the number of failures, but too much preventive maintenance will increase the total maintenance cost, while insufficient maintenance activities will increase the failure rate and increase the equipment failure maintenance cost. Therefore, the start time, time interval, and times of preventive maintenance should be scientifically and reasonably arranged to minimize the total maintenance cost and maximize the reliability of the test chamber.

The multi-objective optimization model of reliability maintenance cost is as follows, considering the multiple objectives of keeping high reliability and corresponding cost control in a low range in the whole equipment operation cycle:

Find:

$$
N, t_{i}
$$

Max:

$$
R\left(N, t_{i}\right)=\frac{\sum_{i=1}^{N+1} \int_{0}^{t_{i}} \exp \left(-\int_{0}^{t} \lambda(t) d t\right) d t}{\sum_{i=1}^{N+1} t_{i}}
$$

Min:

$$
C\left(N, t_{i}\right)=\sum_{i=1}^{N+1} \int_{0}^{t_{i}} c_{c m} \bullet \lambda(t) d t+\sum_{i=1}^{N} c_{p m}\left(t_{i}\right)+\sum_{i=1}^{N} c_{p t}
$$


s.t.

$$
\sum_{i+1}^{N+1} t_{i} \leq T
$$

Therefore, the maintenance strategy of the environmental test chamber with reliability as the center and preventive maintenance as the means is constructed as a multi-objective optimization model to achieve cost control while ensuring reliability to meet the requirements.

\section{EXAMPLES}

\section{A. Failure data of environmental test chamber}

For the environmental test chamber, operation data and troubleshooting data are more valuable. Generally, the amount of data used by a single piece of equipment is small, which is not enough to support the reliability analysis of the environmental test chamber. Therefore, this paper uses the maintenance data of several environmental test chambers from an equipment maintenance company for analysis.

In the analysis of failure data of environmental test chamber, researchers should start with the possible failure of environmental test chamber; Secondly, the cause of the failure phenomenon is studied, which system or component of the environmental test chamber is damaged; Finally, focus on the components and systems with high failure rate, reasonably arrange the spare parts management, and formulate the corresponding maintenance strategy, to meet the requirements of low maintenance cost under the condition of high equipment reliability.

According to the original data of the maintenance records of the environmental test chamber, the failure maintenance data of the environmental test chamber for analysis and research are obtained. The main failure equipment includes the following categories: electrical failure, humidification failure, refrigeration failure, control failure, waterway failure, structural failure, etc., as shown in Figure 2.
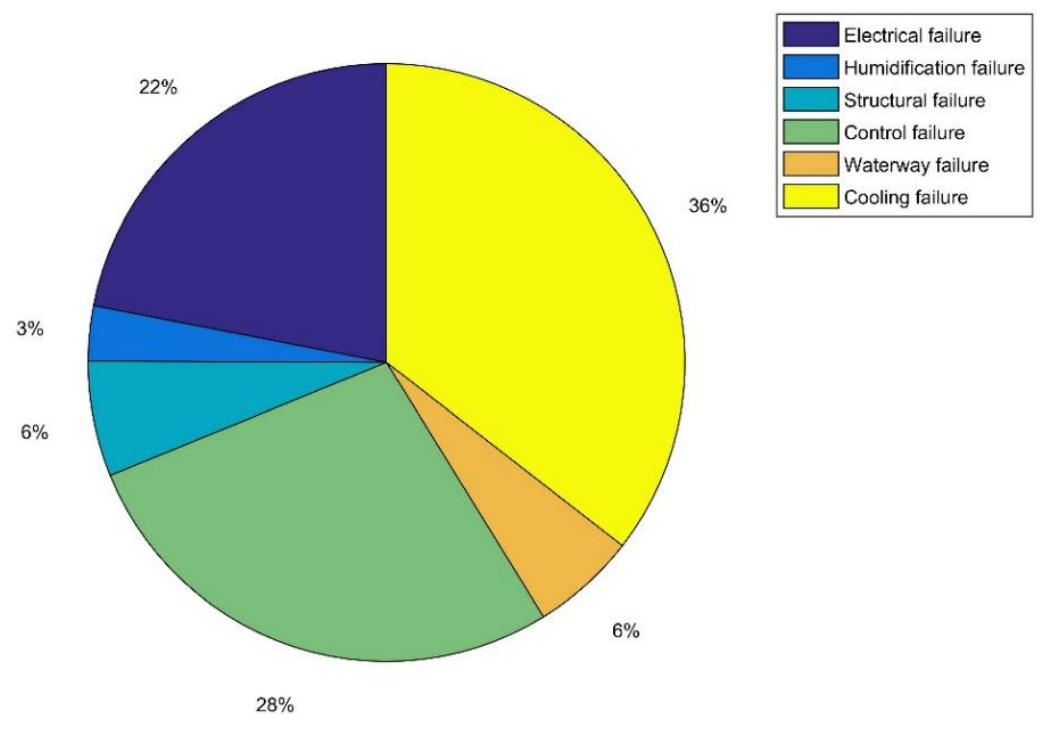

Fig. 2 Failure frequency statistics of each subsystem of the environmental test chamber

For the reliability analysis of the environmental test chamber, its reliability can be easily analyzed after understanding its fault distribution [9].

According to the existing failure maintenance data of the environmental test chamber, the failure data of 3 test chambers of equipment model $02066502-4$ is taken as a case study. The probability density function is fitted by observing the time between failures. Firstly, the failure data of the environmental test chamber are grouped. Use the following empirical expression (4) to determine the number of groups $k$ :

$$
k \geq 1+3.32 \lg N
$$

where $N$ is the total number of failures in the environmental test chamber, and the number of failures in the failure data is 38 .
According to equation (4), the number of groups $k$ is 7 [10]. Specific fault data grouping is shown in Table 1.

According to the grouping of the fault data of the environmental test chamber, the empirical distribution fitting of the fault data is first carried out, as shown in Fig 3.

The empirical distribution function of the environmental test chamber is defined as:

$$
F(t)=P(T \leq t)
$$

where $t$ is the time between failures of the environmental test chamber and $T$ is the overall time between failures. The empirical distribution function of time between failures of the environmental test chamber is obtained as follows: 
$F_{n}(t)\left\{\begin{array}{c}0, t<t_{1} \\ i / n, t_{i} \leq t<t_{i+1}, i=1 \ldots n \\ 1, t \geq t_{n}\end{array}\right.$

When the number of samples is large, the empirical distribution of sample observations can be used to approximate the actual fault distribution. Since the derivative of fault distribution is its probability density function, the type of fault distribution can be preliminarily determined according to the shape of the probability density function [11].

Fig 3. Empirical distribution fitting of environmental test chamber fault data

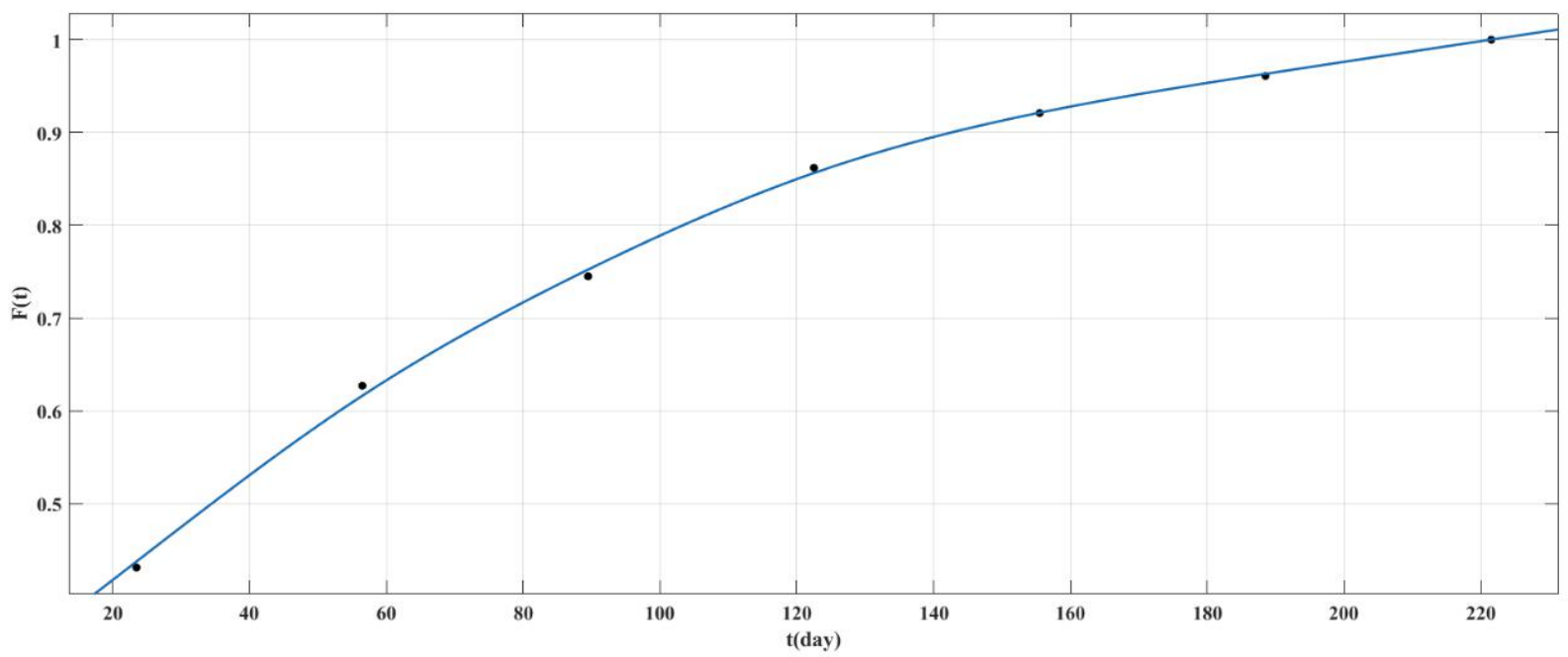

Table 1. Fault Data Grouping and Frequency Distribution

\begin{tabular}{ccccccc}
\hline Group & Left interval & Median & Right interval & Frequency & $\begin{array}{c}\text { Frequency } \\
\text { rate }\end{array}$ & $\begin{array}{c}\text { Cumulative } \\
\text { frequency }\end{array}$ \\
\hline 1 & 7 & 23.5 & 40 & 22 & 0.431 & 0.431 \\
2 & 40 & 56.5 & 73 & 10 & 0.196 & 0.627 \\
3 & 73 & 89.5 & 106 & 6 & 0.117 & 0.745 \\
4 & 106 & 122.5 & 139 & 6 & 0.117 & 0.862 \\
5 & 139 & 155.5 & 172 & 3 & 0.058 & 0.921 \\
6 & 172 & 188.5 & 205 & 2 & 0.039 & 0.961 \\
7 & 205 & 221.5 & 238 & 2 & 0.039 & 1.000 \\
\hline
\end{tabular}

According to Fig 3, it can be observed that the empirical distribution function of the time between failures of the environmental test chamber shows the shape characteristics, without inflection point and convex. which conforms to the shape characteristics of Weibull distribution and exponential distribution, and the exponential distribution can be regarded as a special Weibull distribution. Therefore, it is assumed that the fault distribution is Weibull distribution. The least-square method is selected to solve the Weibull parameter. According to the correlation coefficient method to test the final distribution effect, the failure interval distribution type of environmental test chamber is obtained.

The two-parameter Weibull distribution satisfies most of the fault distribution rules, so the two-parameter Weibull distribution is usually used to study the fault distribution in practical engineering.

Firstly, the parameter estimation of the Weibull distribution is carried out. The linear regression equation of one-dimensional is as follows:

$$
y=A+B x
$$

For the two-parameter Weibull distribution, the linear transformation of the distribution function can be obtained as follows:

$$
\begin{gathered}
y=\ln \ln \left(\frac{1}{1-F(t)}\right) \\
x=\ln t \\
A=-\beta \ln \alpha, B=\beta \\
\hat{F}\left(t_{i}\right) \approx \frac{i-0.3}{n+0.4}
\end{gathered}
$$

According to the least square method: 


$$
\begin{aligned}
& \hat{B}=l_{x y} / l_{x x} \\
& \hat{A}=\bar{y}-\hat{B} \bar{x}
\end{aligned}
$$

among them:

$$
\begin{aligned}
& l_{x x}=\sum_{i=1}^{n}\left(x_{i}-\bar{x}\right)^{2}=\sum_{i=1}^{n} x_{i}^{2}-n \bar{x}^{2} \\
& l_{y y}=\sum_{i=1}^{n}\left(y_{i}-\bar{y}\right)^{2}=\sum_{i=1}^{n} y_{i}^{2}-n \bar{y}^{2}
\end{aligned}
$$

two parameters of Weibull distribution are estimated by the above formula:

$$
\begin{gathered}
\alpha=70.892 \\
\beta=1.285
\end{gathered}
$$

the linear regression equation is as follows:

$$
y=1.285 x-5.463
$$

the correlation coefficient of the Weibull distribution is tested for the parameters obtained, and the correlation test shows that

$$
r=0.971 \text {. }
$$

D-test is used to arrange the failure data of the environmental test chamber. According to the hypothesis, $\mathrm{F}\left(\mathrm{x}_{\mathrm{i}}\right)$ corresponding to each data is calculated and compared with the empirical distribution [12]. The maximum absolute value of the difference is selected as the observed value of the test statistic and compared with the critical value. The observed values are obtained from the fault data and empirical distribution:

$$
D_{n}=0.1101
$$
formula:

Select significant level $a=0.1$, According to the empirical

$$
D_{n, \alpha}=1.22 / \sqrt{n=} 0.169>D_{n}
$$

Therefore, the original assumption is accepted, that is, the time between failures of 3 environmental test chambers follows the two-parameter Weibull distribution.

The time between failures PDF of this series of environmental test chambers is as follows:

$$
f(t)=0.0174 *\left(\frac{t}{70.218}\right)^{0.285} \exp ^{-\left(\frac{t}{70.218}\right)^{1.285}}
$$

the failure rate function is as follows:

$$
\lambda(t)=0.0174 *\left(\frac{t}{70.218}\right)^{0.285}
$$

The failure rate function is: the calculated Weibull distribution is fitted with the actual data, and the CDF comparison chart is shown in Fig 4.

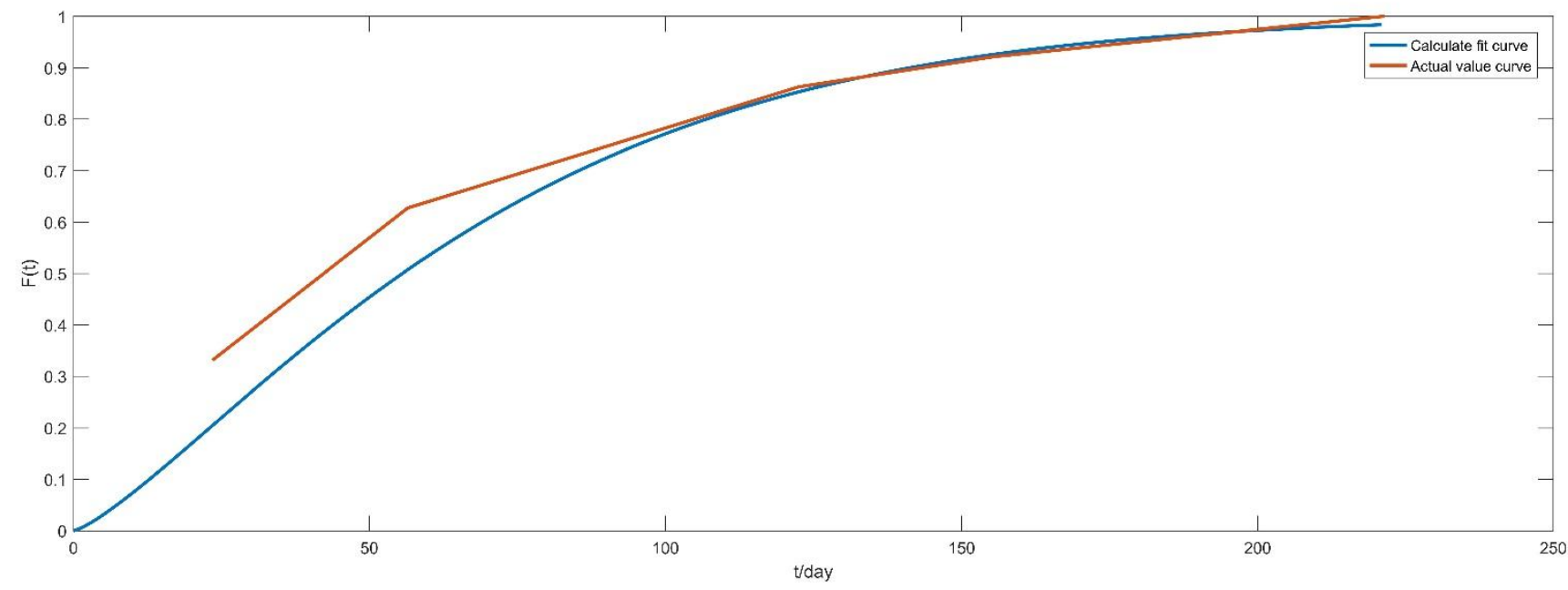

Fig 4. Weibull distribution probability distribution and empirical distribution fitting diagram

\section{B. Particle swarm optimization}

Particle swarm optimization [13] is a kind of swarm intelligence algorithm. Compared with the evolutionary algorithm, it has the following advantages: it retains the global search of the population, has less parameter speed position, and has fast convergence speed. In an n-dimensional search space, the particle update formula is as follows:

$$
\bar{v}_{i}(t+1)=w \bar{v}_{i}(t)+\varphi_{1} * r_{1}\left(\bar{p}_{i}(t)-\bar{x}_{i}(t)\right)+\varphi_{2} * r_{2}\left(\bar{g}_{i}(t)-\bar{x}_{i}(t)\right)
$$

$$
\bar{x}_{i}(t+1)=\bar{x}_{i}(t)+\bar{v}_{i}(t+1)
$$

In the above formula, $p_{i}(t)$ represents the best position of each particle in the search process, which is expressed as the "self-cognition" of particles. $g_{i}(t)$ represents the individual particle with the best position and speed in the search process, which is expressed as the "social part" of the particle. Other particles should keep flying towards the best particle. $w$ represents the inertia factor used to adjust the searchability in the search process; $\varphi_{1}$ is the individual learning factor; $\varphi_{2}$ is the social learning factor; $r_{1}$ and $r_{2}$ are random numbers in the range $[0,1]$.

Multi-objective particle swarm optimization (MOPSO) $[14,15]$ has individual and global optima in the optimization process, and the optimal solution is obtained by iterative optimization along this direction. The specific process is as Fig 5.

(1) Parameter setting, initialization of particle population, calculation of fitness value, and speed. 
(2) Judge the advantages and disadvantages of particles to determine the dominant solution and not only the matching solution.

(3) Build an external archive and expand the non-dominated solution [16].

(4) Choose the comparison between the individual best and the historical best to determine the "self-cognition" sample.
(5) Select the globally optimal particle. Roulette is used to selecting the particles in the mesh as the global optimal samples.

(6) Update particle position and velocity.

(7) Determine whether the termination condition is satisfied, and terminate the operation if the number of iterations is satisfied.

Fig 5. Flow chart of multi-objective particle swarm optimization algorithm

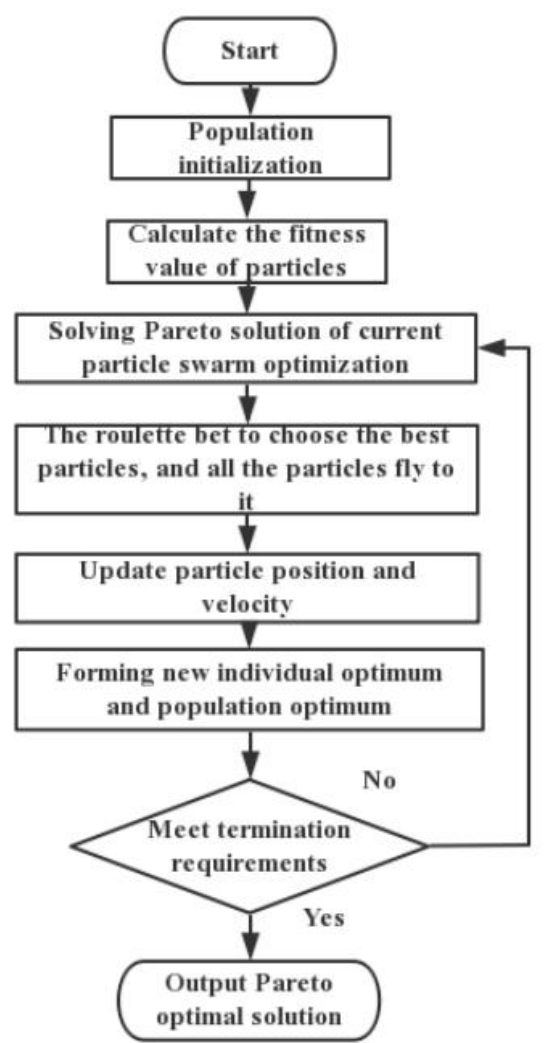

\section{Multi-OBJECTIVE OPTIMIZATION OF MAINTENANCE STRATEGY FOR ENVIRONMENTAL TEST CHAMBER}

In this paper, the fault data of the environmental test chamber is analyzed as an example, and the maintenance strategy of the environmental test chamber is studied. After fitting the fault distribution of the fault data of three test chambers, the assumption of Weibull distribution is obtained, and the specific cost data is taken as an example for analysis. The maintenance cost unit is CNY(Chinese Yuan). The specific example values are shown in Table 2.

Table 2. Numerical analysis of an example

\begin{tabular}{|c|c|c|c|c|c|c|}
\hline $\begin{array}{c}\text { Equipment } \\
\text { running time } \mathrm{T} \\
\text { (day) }\end{array}$ & $\begin{array}{l}\text { Minimum time of } \\
\text { maintenance } \\
\text { interval (day) }\end{array}$ & $\begin{array}{c}\text { Minimum } \\
\text { maintenance cost } \\
\mathrm{C}_{\min }(\mathrm{CNY})\end{array}$ & $\begin{array}{c}\text { Preventive } \\
\text { maintenance cost } \\
\mathrm{C}_{\mathrm{pm}}(\mathrm{CNY})\end{array}$ & $\begin{array}{l}\text { Loss of sequential } \\
\text { maintenance } \\
\text { downtime }(\mathrm{CNY})\end{array}$ & $\begin{array}{c}\text { shape parameter } \\
\mathrm{m}\end{array}$ & $\begin{array}{c}\text { Scale parameter } \\
\eta\end{array}$ \\
\hline 600 & 10 & 3000 & $500+0.2 * \mathrm{t}_{\mathrm{i}}$ & 1000 & 1.283 & 70.218 \\
\hline
\end{tabular}

The above data, according to the relevant suggestions of enterprises and maintenance engineers, conform to the real environmental test chamber operation reliability and cost data.

In this paper, the multi-objective particle swarm optimization algorithm is used to solve the reliability maintenance cost multi-objective optimization model of the environmental test chamber. The initial population number is 50 , the number of iterations is 100 , the initial weight is 0.5 , the non dominated population number is 15 , and the weight attenuation factor is 0.99 .

According to the reliability maintenance cost model of the environmental test chamber, the decision variables are periodic preventive maintenance times $N$ and maintenance interval $t_{i}$. Therefore, according to the idea of the model, the corresponding time interval $t_{i}(i=1, \ldots N)$ is obtained by calculating the number of maintenance from $N=1$, and then increasing the number of maintenance in turn. The Pareto solutions of different maintenance times and time intervals of 
the reliability maintenance cost model corresponding to the maintenance times and time intervals are obtained [17]. Through the solutions containing the information of maintenance times and time intervals, the maintenance strategies of the environmental test chamber are formulated for reference.
MOPSO is used to solve the problem, and $N=5$ maintenance times are selected as an example to illustrate the solving process of reliability maintenance cost multi-objective particle swarm optimization algorithm. The following is a randomly generated distribution map of the primary population.

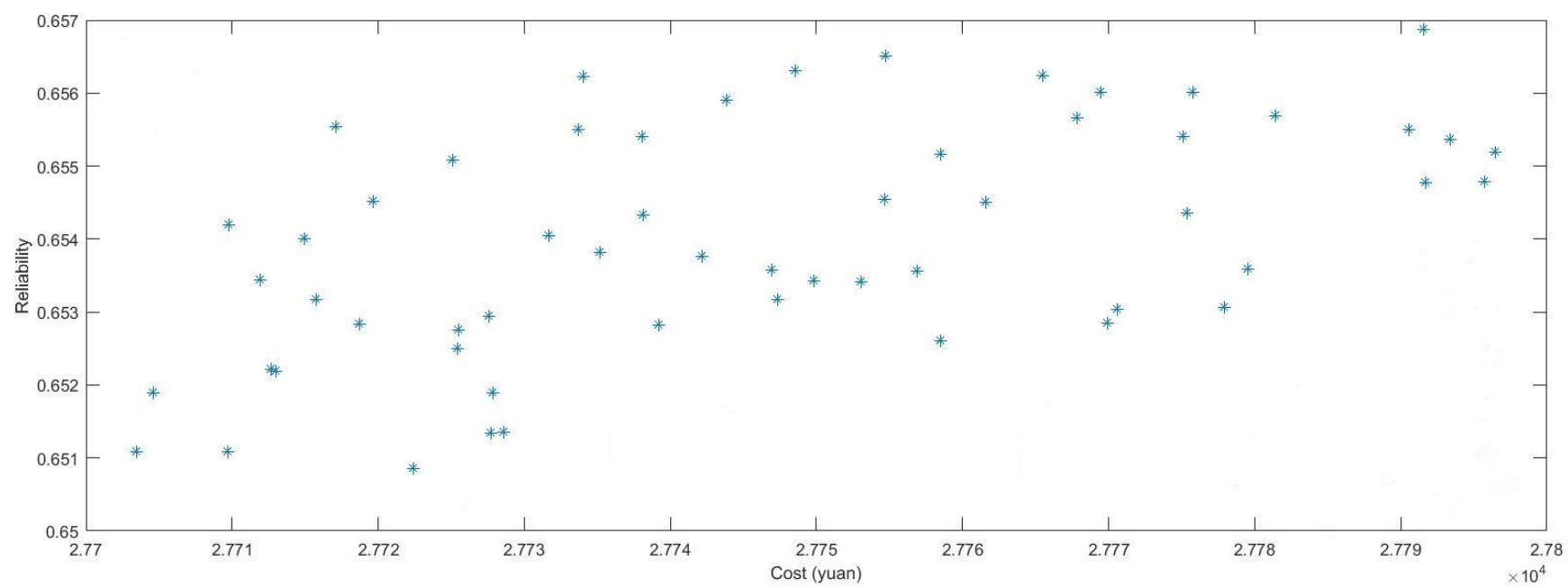

Fig 6. Randomly generated primary population distribution

From the randomly generated population distribution, we can see that the population density is small and widely distributed, which ensures the diversity of particles. The abscissa represents the maintenance cost, and the unit is CNY. The ordinate represents the reliability, and the range is $0-1$.
In this paper, the result of 100 iterations is taken as the final optimization result, and the final reliability cost distribution diagram of $N=5$ solved by a multi-objective particle swarm optimization algorithm is shown in Fig 7.

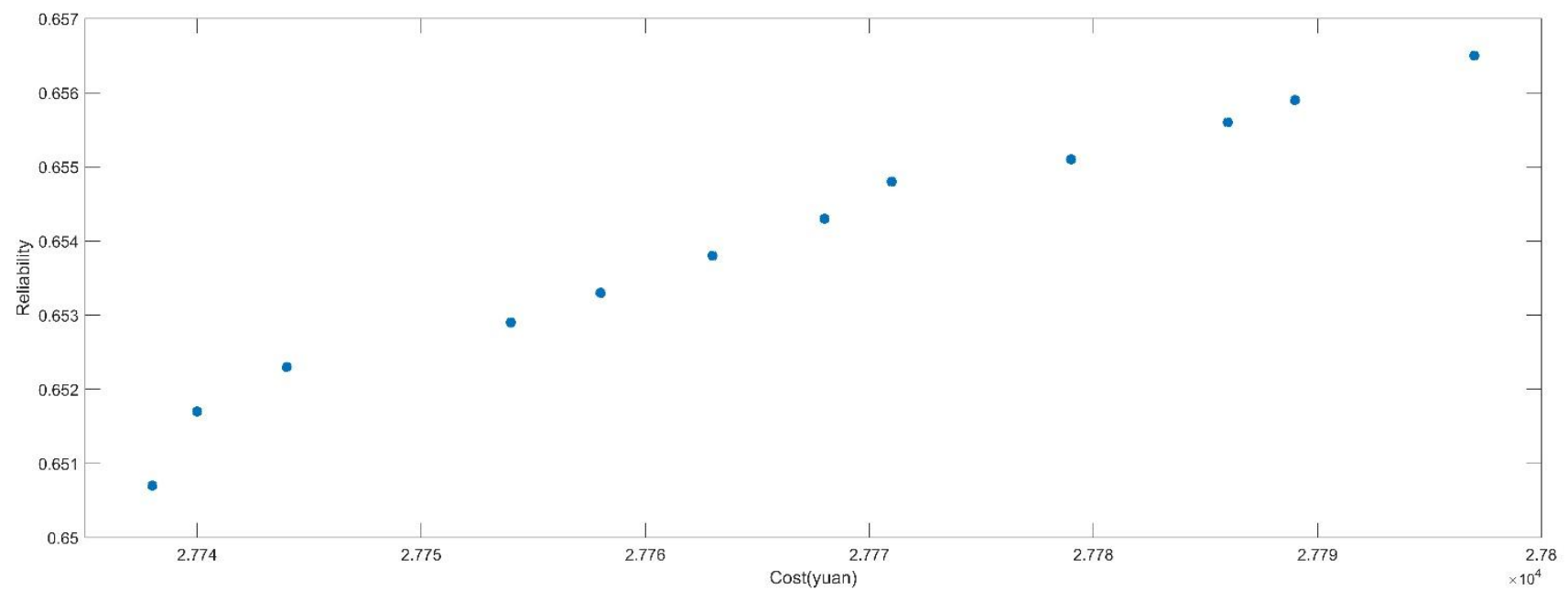

Fig 7. Final optimization results of 5 maintenance times

Because the fault distribution is calculated by the empirical distribution fitting of the actual data, the reliability of the environmental test chamber reaches about 0.83 when the maintenance times are 9 times. With the increase of maintenance times and iterations, the reliability growth is small, and no longer changes significantly, and the cost increases rapidly. The environment test chamber analyzed in this study has a long service life and has been repaired many times. Considering the actual operation situation, the maintenance times in the maintenance strategy are set as 1-9 times by consulting the maintenance engineer. Fig 8 shows the reliability cost optimization results of 9 maintenance times. 


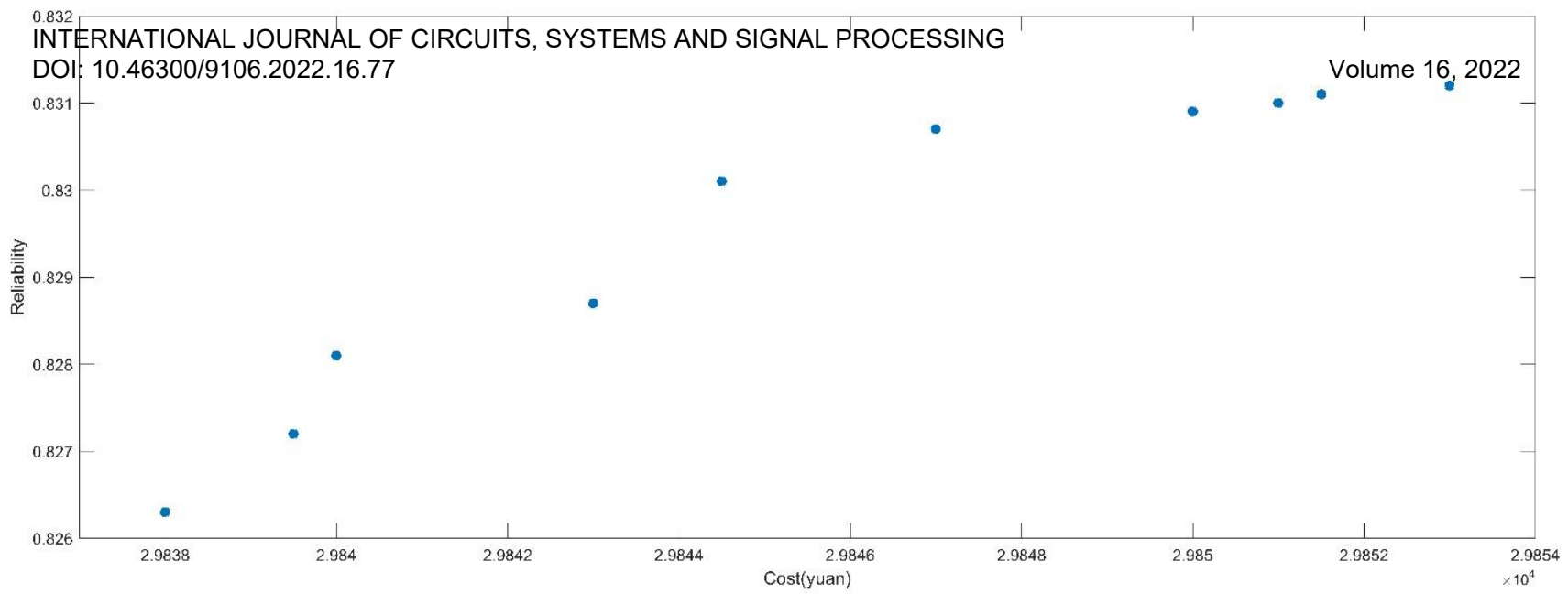

Fig 8. Final optimization results of 9 maintenance times

All the optimization results of 1-9 maintenance times in the maintenance plan are shown in Fig 10. It can be seen that with the increase of maintenance times, the reliability and cost of the environmental test chamber are improved. When the number of maintenance is 1 to 3 , the change of reliability is small, but the cost increases more. The maintenance plan does not meet the actual maintenance requirements of the environmental test chamber, and the corresponding reliability is low, which leads to the environmental test chamber being in the state of under maintenance. Therefore, the maintenance plan with 1-3 times of maintenance is eliminated.

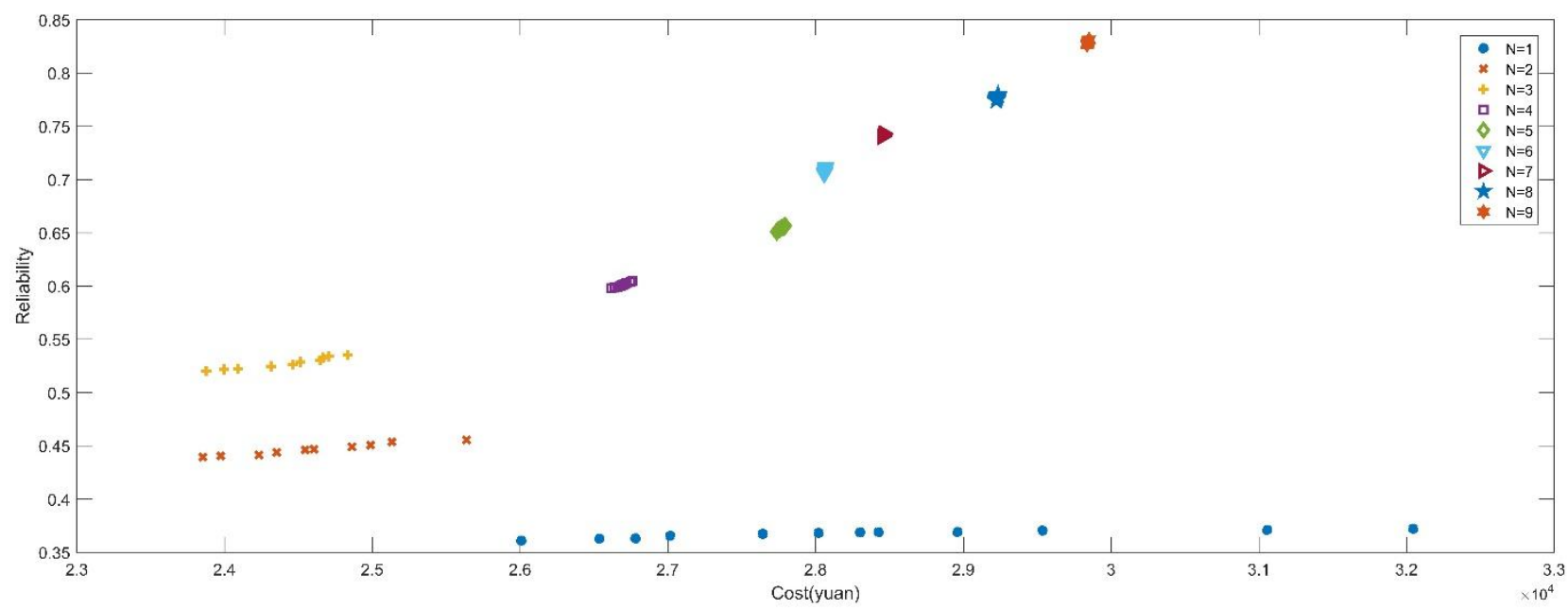

Fig 9. Maintenance plan optimization results

With the optimization results of the reliability cost multi-objective model of the maintenance strategy, how can the decision-maker choose a maintenance plan to meet their own needs? If the decision-making is to consider the maintenance cost, such as controlling the cost range, the lowest cost in the planning model with reduced maintenance times should be selected; If the reliability is considered to meet a certain range, it is necessary to select the optimization result point with more maintenance times and higher cost. For example, if the reliability is kept at about 0.6 and the maintenance cost is less than $27000 \mathrm{CNY}$, four maintenance times can be selected, as shown in Fig 10, and the maintenance interval of the lowest cost point a $(27738,0.6507)$ is $(78,83,87,98,130$ days $)$; If the reliability is greater than 0.7 and as high as possible, the maintenance times can be more than 7 times. Take 8 times of maintenance as an example, the final maintenance plan selection point B $(29235,0.7801)(53,49,50,49,53,60,60,73$ days) is determined, as shown in Fig 11. In general, the final Pareto solution represents different maintenance plans. How to choose a reasonable plan is up to the decision-maker. 


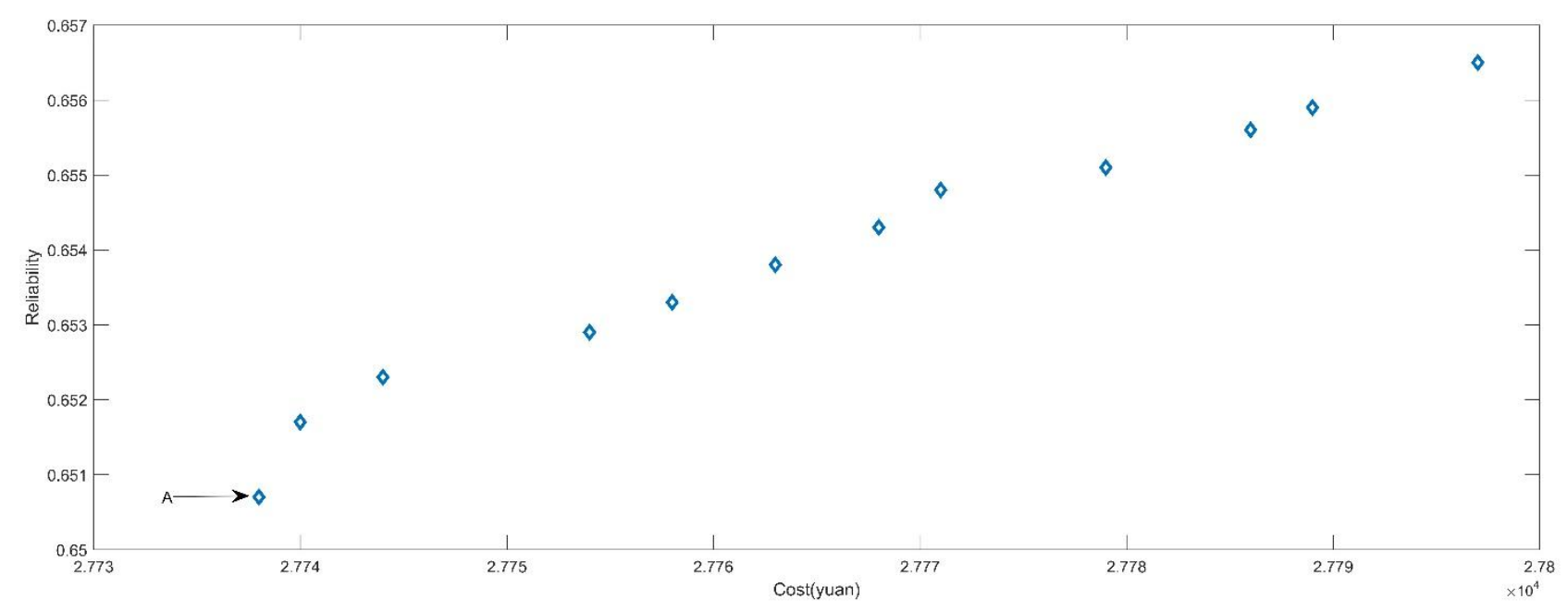

Fig 10. Minimum maintenance planning cost point A

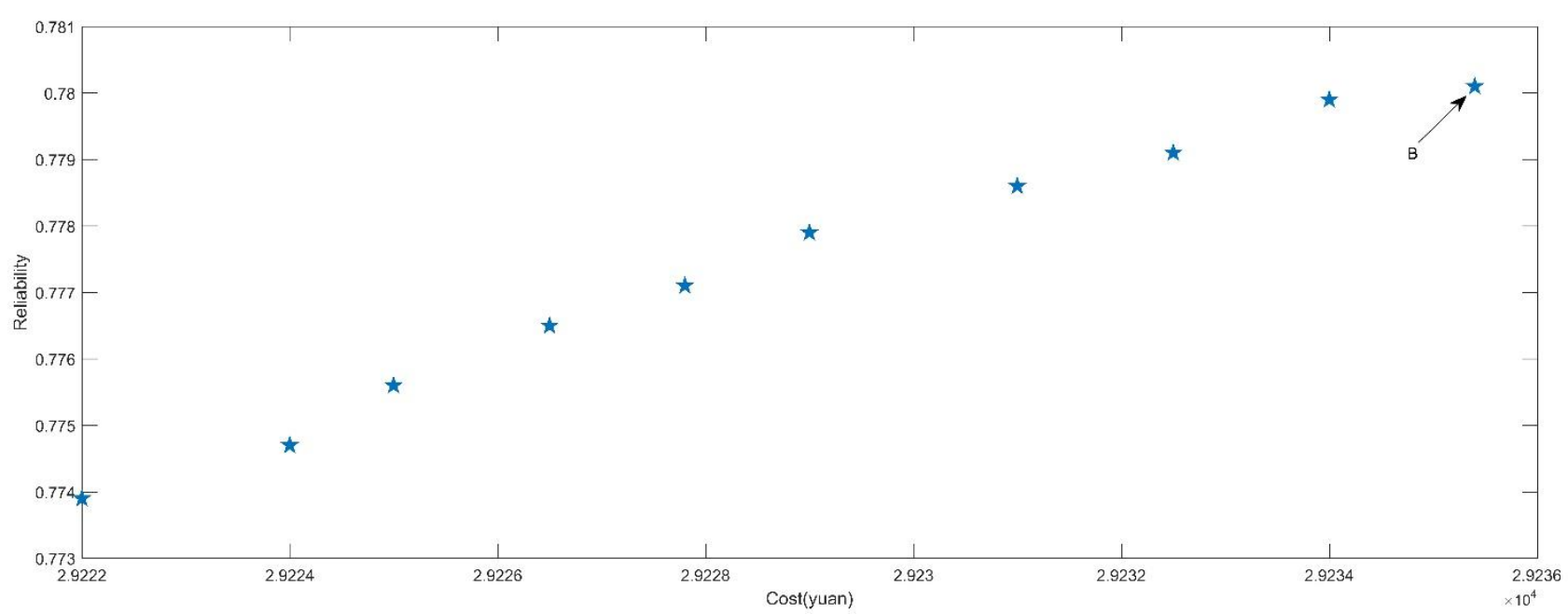

Fig 11. Maximum reliability point B of maintenance plan

In the process of using an environmental test chamber, a maintenance plan is selected and made according to different test scenarios. If the test period is short, the cost can be chosen as the center; If the test cycle is long, to avoid test interruption, a maintenance plan should be made based on the principle of high reliability.

\section{CONCLUSION}

The traditional maintenance methods of the environmental test chamber mostly adopt post-maintenance and carry out maintenance activities after the failure, which can not meet the current process of reliability tests. Therefore, this paper chooses to develop the periodic preventive maintenance strategy with reliability as the center. Through the analysis of the fault fitting distribution of the actual environmental test chamber fault maintenance data, it is determined that the fault distribution of the environmental test chamber meets the double parameter Weibull distribution. Taking the failure rate function as the intermediate, a multi-objective optimization model of reliability and maintenance cost of the environmental test chamber is established. Combined with the actual operation data and expert advice, the numerical value of the example is determined, and the multi-objective optimization problem is solved by using the MOPSO algorithm. A group of maintenance plan solutions are generated, which provides a reference for decision-makers to make maintenance plans. For the maintenance strategy of the environmental test chamber, the multi-objective optimization model of reliability maintenance cost can be calculated by other algorithms, such as a genetic algorithm. Different algorithms can be selected for the solution, and the results are compared to select a more suitable algorithm for the solution. 


\section{REFERENCES}

[1] G. L. Liu, "Use and maintenance of high and low-temperature humid heat test equipment," Science and Technology Information, vol. 001, no. 008, pp. 119-120, 2010.

[2] C. Gu, L. Zhai, W. Gu, Y. Sun, C. Han, X. Zhou and W. Niu, "System analysis and reliability analysis for environmental test chambers," International Journal of Circuits, Systems, and Signal Processing, vol. 14, no.1 pp. 1115-1128, 2021.

[3] R. Kang, "Introduction to reliability and maintainability engineering," Industrial Engineering and Management, no. 3 , pp. 66-68, 2010.

[4] Y. H. Wang, J. Y. Jiao, and B. W. Yu "Research on preventive maintenance strategy of equipment based on particle swarm optimization algorithm," Firepower and Command Control, vol 2, no.1 pp. 1-6,2021.

[5] Q. Chen, "Research on equipment maintenance optimization based on reliability," Tianjin University, no. 3, pp. 15-17, 2008.

[6] Y. Xiao, X. Han, J. Sun, and J. Zhang, "Research on equipment maintenance strategy aiming at maximum success probability," Journal of Weapon Equipment Engineering, vol. 40, no.256, pp. 139-144, 2019.

[7] Q. Wang, X. Jia, Z., "Reliability modeling and preventive maintenance strategy of equipment system based on competitive failure in wartime," System Engineering and Electronic Technology, vol. 041, no. 010, pp. 2392-2400, 2019.

[8] X. Xiao, D. Xiao, J. Lin, "Overview of multi-objective optimization problems," Computer Application Research, vol. 28, no. 003, pp. 805-807, 2011.

[9] D. Marquez, M. Neil, and N. Fenton, "Improved reliability modeling using Bayesian networks and dynamic discretization," Reliability Engineering \& System Safety, vol. 95, no. 4, pp. 412-425, 2010.

[10] J. Fan, H. Wang, L. Zhang "Research on Reliability Modeling and evaluation of CNC grinding machines," Manufacturing Technology and Machine Tools, vol.01, no. 6, pp. 94-97, 2016.

[11] H. Zhang, Y. Jia, and G. Zhou, "Research on the distribution model of time between failures in $\mathrm{CNC}$ systems," Journal of the Harbin University of Technology, vol. 37, no. 002, pp. 198-200, 2005.

[12] G. Zhao, "Research on p-value correction method of the statistical hypothesis test, "Beijing University of Technology, no. 3, pp.23-24, 2014.

[13] J. Kennedy and R. Eberhart, "Particle swarm optimization," In Icnn95-international Conference on Neural Networks, 2002

[14] C. Coello, G. T. Pulido, and M. S. Lechuga, "Handling multiple objectives with particle swarm optimization," IEEE Transactions on Evolutionary Computation, vol. 8, no. 3, pp. 256-279, 2004.

[15] C. Coello, D. Ing, and M. S. Lechuga, "Mopso: A proposal for multiple objective particle swarm," Proceedings of IEEE International Conference On Neural Networks, Computer Society, pp. 1051-1056, 2003.
[16] Rui. Leng, L. Yuan, and Y. Liu, "Research on improved multi-objective particle swarm optimization algorithm based on optimal grid distance," Journal of Zunyi Normal University, vol. 20, no. 05, pp. 104-108, 2018.

[17] S. Huang, Y. Wang, and Z. Ji, "Dynamic multi-fuel economic environment load distribution based on multi-objective particle swarm optimization algorithm," Journal of Control and Decision Making, vol. 33, no. 007, pp. 1255-1263, 2018.

Xianwen Zhou is an engineer of the Key Laboratory of Transport Industry of Marine Technology and Control Engineering, Shanghai Maritime University, Shanghai, China. He mainly engaged in reliability test research, product testing, ship and port automation design research, and reliability test equipment maintenance.

Chaoyang Gu received the B.E. degrees from Zhoukou Normal University, Zhoukou, China, in 2016. He is currently pursuing an M.S. degree from Shanghai Maritime University, Shanghai, China. His research direction is the reliability analysis of environmental test chambers.

Yuyu Sun is a senior engineer at Jiangsu Automation Research Institute, Lianyungang, China, He engaged in research work in laboratory equipment management.

Chengjing Han is the chief engineer of Shanghai Sizhong Experimental Equipment Co., Ltd., Shanghai, China. He is engaged in the commissioning, maintenance, and repair of environmental test chambers with 16 years of experience.

Wei Gu received the B.E. and Ph.D. degrees from Shanghai Maritime University, Shanghai, China, in 1982 and 2008, respectively.

Since 1982, he has been a Teacher at Shanghai Maritime University, where he has been a Professor, since 1997, and is currently the Director of the Key Laboratory of Transport Industry of Marine Technology and Control Engineering. His research interest includes marine information control technology.

Wangqiang Niu received the B.E. degree from Xi'an Aerotechnical College, Xi'an, China, in 1998, the M.E. degree from Northwestern Polytechnical University, Xi'an, in 2004, and the Ph.D. degree from Shanghai Jiao Tong University, Shanghai, China, in 2008.

From 2013 to 2014, he was a Visiting Lecturer with McMaster University, Hamilton, ON, Canada. Since 2008, he has been a Lecturer with Shanghai Maritime University, Shanghai, where he has been an Associate Professor, since 2017. His research interests include wireless power transfer, control of marine equipment, and prognostics and health management.

\section{Creative Commons Attribution License 4.0 (Attribution 4.0 International, CC BY 4.0)}

This article is published under the terms of the Creative Commons Attribution License 4.0 https://creativecommons.org/licenses/by/4.0/deed.en_US 